\title{
HYBRID CLASSIFIER TO CLASSIFY THE FINGER NAIL ABNORMALITIES
}

Thahira Banu.V

Research Scholar, Bharathiar University, and

Assistant Professor, Sri Krishna Arts and Science, College, Coimbatore, Tamil Nadu. India

\author{
Dr. M. Renuka Devi \\ Head, BCA Department, \\ Sri Krishna Arts and Science College, \\ Coimbatore, Tamil Nadu. India
}

\begin{abstract}
Nail diagnosis is a method to predict the possibilities of organ failures and various systemic diseases. Nail abnormalities are considered as the signs of certain diseases in traditional medicines such as Siddha Medicine, Ayurveda, Yunani and Chinese medicine etc. In this paper, the performance of existing techniques such as SVM classifier and KNN classifiers are compared with the proposed method. The metrics precision, recall, F-measure and accuracy are calculated and compared. The 100 images had taken for study and the proposed novel segmentation method gives the best accuracy. The experiment uses 480 (increase the dataset) images of eight types of abnormalities. $70 \%$ of images were used for training and $30 \%$ of images were used for testing. (Discuss the performance measure)
\end{abstract}

Keywords - Finger nail image analysis; KNNwithSVM classifier; KNN classfier; SVM classfier; finger nail disease prediction;

\section{INTRODUCTION}

In medical Imaging diagnosis methods are used to measure the severe of the disease and for further treatment. Some may invasive and others may noninvasive techniques. Mostly, the diseases can be identified by symptoms and external appearances. Generally, the medical practitioners check the eye, tongue, nail and palm abnormalities as the indication of the diseases and they prefer further diagnosis method or investigations. Here, the nail diagnosis method is considered for the research work using medical image processing method, which contributes to tele-medicines, mass-screening and teledermatology fields. Nail abnormalities are the signs of the systemic diseases happening in the human body. The abnormalities of finger nails are the signs for various disease and organ failures or for severe systemic diseases. Nail abnormalities are classified by it color, texture, size and shape of the nail. The color and texture of the thumb finger nail plate has considered for our research work,

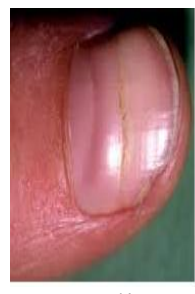

Beau's lines Lunulae
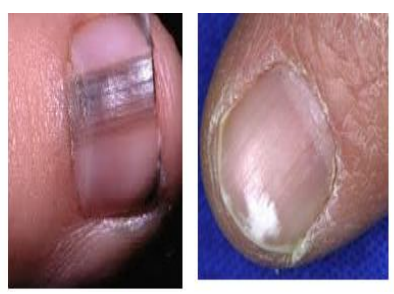

Vertical lines

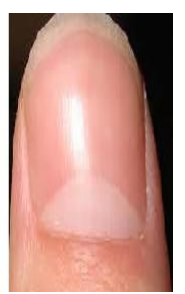

White patches

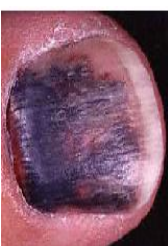

Black Nail colored

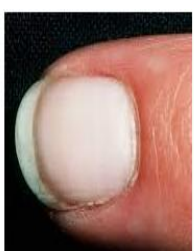

White Nail

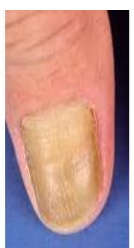

Greenish

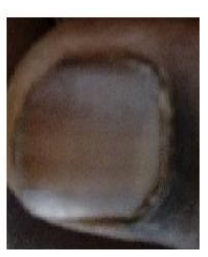

Gray
Fig-1 Nail Abnormalities

There are eighteen types of abnormalities are possible in finger nails. The types of abnormalities are considered for the research work and some of the abnormalities are listed as follows:

(1) Horizontal ridges or Beau's Lines: Injury, infection, mal-nutrition; (2) Infected nails (red, tender, swollen, pus): Bacterial or yeast infection; (3) Overlarge moons (Lunula): Overactive thyroid, genetics, self-induced trauma (habit tick). No Lunula: Underactive thyroid, genetics; (4) ColorlessWhite nails: May indicate anemia (5) Red or dark pink: poor peripheral circulation; (6) Bluish nails: Blood may not be receiving adequate oxygen due to respiratory disorders, cardiovascular problems; (7) Yellowish: fungus, diabetes, psoriasis, use of tetracycline, or heredity; (8) Half white / half pink: fungal infection or kidney disease; (9) Small white patches: a sign of injury to the nail matrix, vitamins and minerals deficiencies; (10) Purple or black: trauma or vitamin B12 deficiency; (11) Brown or 
black streak that begins at the base of the nail and extends to its tip could be a diagnostic clue to a potentially dangerous melanoma.

In this paper, nail image classification has done with the proposed algorithm and compared with the existing algorithms. Section I describe the background truth of the nail plate abnormalities which are used as signs for various diseases and organ failures. Section II narrates how various features and classifiers can be used to extract and classify the diseased areas. Section III elucidates the proposed classifier and Section IV derives the results and discussions.

\section{LITERATURE REVIEW}

Feature extraction is an important process in image analysis, computer vision and pattern recognition based applications. It is mainly used for dimensionality reduction and to classify with high accuracy. Feature means the mathematical information of an image, which is labelled after the segmentations. The characteristics of an image can be stored into a feature vector (Let say $\mathrm{V}=(\mathrm{v} 1, \mathrm{v} 2, \ldots \mathrm{vn}))$ which is known as Feature extraction. Textures can be defined as spatial variation in pixel intensities (gray values).

The texture features Entropy, Correlation, Homogeneity and contrast are extracted by GLCM (Gray Level Co-occurrence Matrix). The basis of GLCM is assigning the association among two neighboring pixels in single offset as the next order surface. The gray values associations in a result image are altered into the co-occurrence matrix by a specified kernel mask $3 \times 3,5 \times 5,7 \times 7$ and so forth. In the alteration from the image onto the co-occurrence matrix, the neighboring pixels in 0 degree orientation can be used. It contains information of the position of pixels having related gray level values (Dr.K.Ramesh Babu et al, 2013) [6]. Entropy is evaluated of unpredictability that is used to describe the texture or surface of the input image. Its value will be highest when all the elements of the co-occurrence matrix are the similar. The contrast is a measure of strength of a pixel and its neighbor over the image.

Classification is a function that distinguishes feature vectors into different classes. It involves two steps: Learning and Classification. In learning or training step, a classifier is constructed by learning from a training dataset and their relevant class labels.
These predefined classes are determined by a class label attribute which serves as a category or class. In classification step, the classifier is used to predict the class labels for given new data which is known as testing. In general, test data are used to estimate the accuracy of the classification rules. The accuracy is the percentage of test data set that are correctly classified by the classifier.

Then randomly only some images are chosen up which are measured to be the centroid of every cluster. Then the distance (i.e, similarity) among the images is establish out by computing the Euclidean distance among the feature vectors of the images being measured. The images are supposed to be parallel if their Euclidean distance is equivalent to the threshold value (user defined value). Thus the related images are grouped into a cluster (here, the cluster is a directory). Having placed all the images into their individual clusters, for every cluster based upon the containing images, novel centroid image is found.

Gulnaz Alimjan et al 2017 [4] proposed a hybrid classifier SV-NN to the remote sensing satellite images. The SVM is applied to the learning phase and KNN is applied to the classify the sample. SVM categories the certain classes by minimum average distance method by Euclidean distance method. The hybrid classifier has compared with conventional classifiers such as KNN, SVM and ANN with the performance metrics overall accuracy and kappa coefficiency.

\section{METHODOLOGY}

The proposed work considers the finger nail image classifciaiton process of Hybird classification algorithm is derived in this portion. This paper introduced a good finger nail image classsifciaton process of Combining KNN with SVM classification algorithm. Previous to classification, the Transitioned Row-Column sorting (TRCSF) denoise filter, Graph Based Threshhistomatch Segmentation (GTHM) segmentation process and the feature is extracted using GLCM feature method. The overall finger nail classification procedure flow diagram is illustrated in figure 2 . 


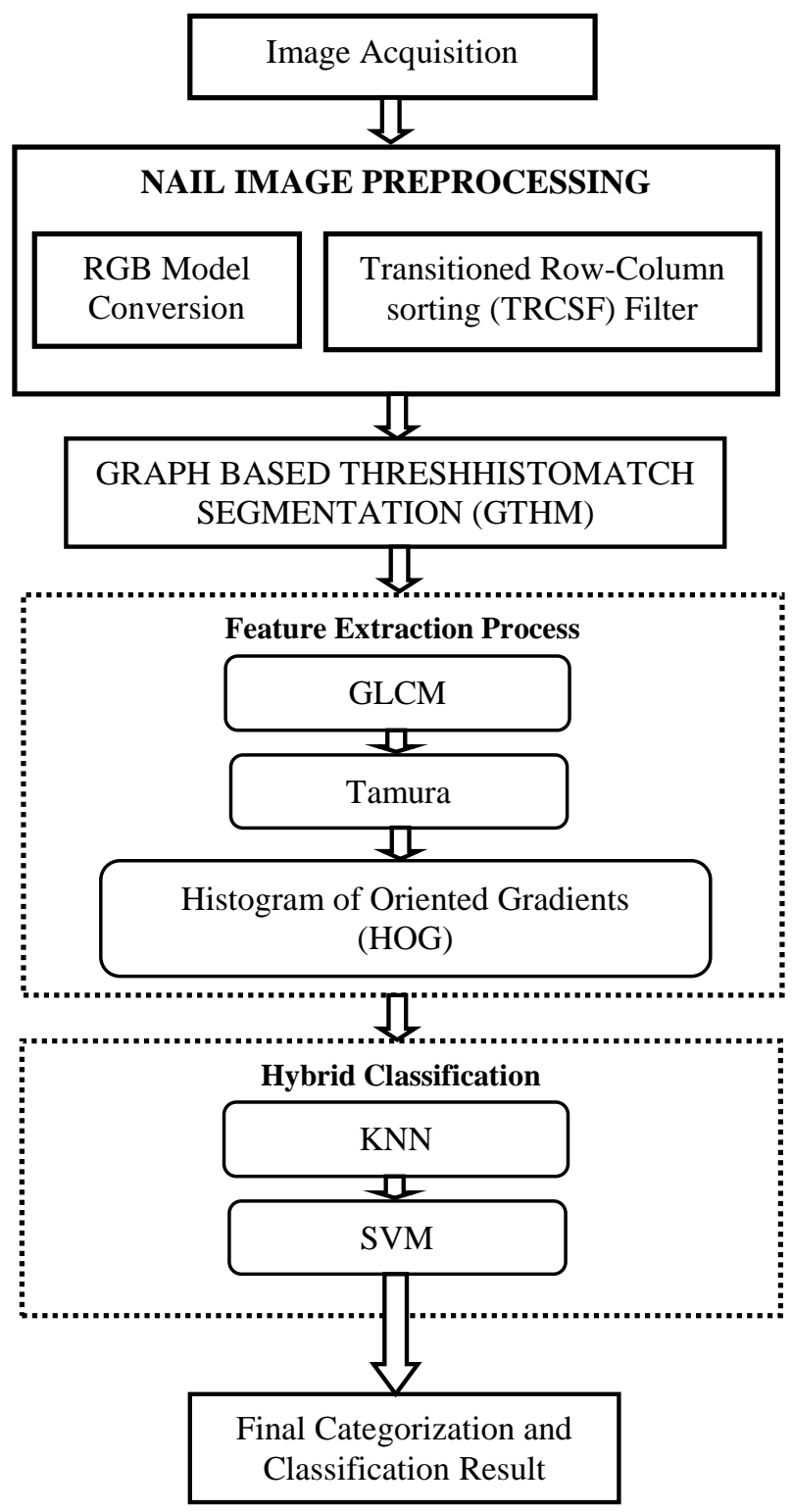

Fig-2: Flow-chart of Proposed work

\section{a) Image Acquisition, Image Preprocessing and GTHM:}

Thahira Banu.V, Dr. M. Renuka Devi, 2020 [2-3], already processed the following techniques: Image Acquisition, Transitioned Row-Column sorting (TRCSF) denoise Filter, Graph Based Threshhistomatch Segmentation (GTHM) for Segmentation. The finger nail images may contain noises thus it reduces the redundant noises to notice the injuries. So, TRCSF filter is executed to get improved the PSNR value and to increase the fineness of the image. After TRCSF de-noising process, segmentation method is completed with GTHM Algorithm can be done with skin detection and threshistomatch algorithm is efficiently using the global and automatic thresholding techniques which enhance the step convolution of the proposed system which segments the disease portions from the

Copyright $(\odot)$ Author denoised image results that are shown in Fig 3. The above three procedures are done before extracting the features of the finger nail images.

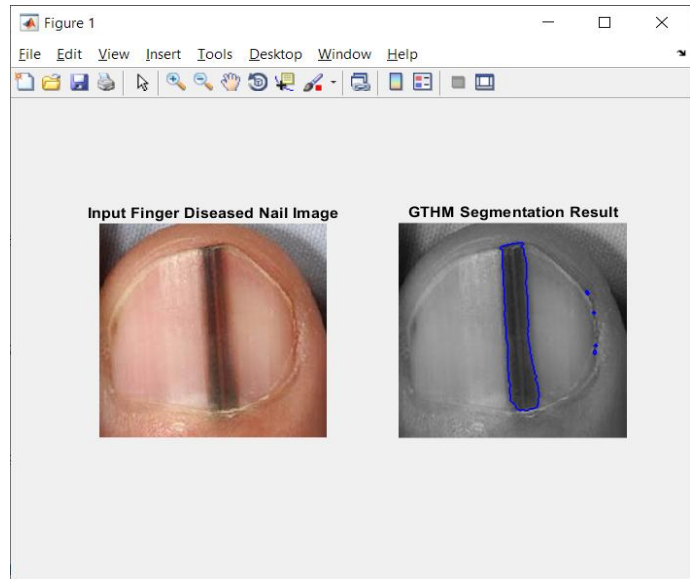

Fig-3: GTHM Segmentation Result

\section{b) Feature Extraction}

After the GTHM segmentation procedure a Gray Level Co-occurrence Matrix (GLCM) Feature based Extraction with contrast, correlation, energy, homogeneity, mean, standard deviation, entropy, RMSE, variance, smoothness, kurtosis, skewness, ID (Image Difference), HOG (Histogram Oriented Gradient) and Tamuraf features are executed. HOG feature descriptor focuses on the disease structure or the disease shape of an object. Tamura features will execute on homogeneous texture images. This paper proposed GLCM based Feature Extraction algorithm to obtain the trained features which will be used for Hybrid Classification procedure. The feature extraction results are shown in Figure 4.

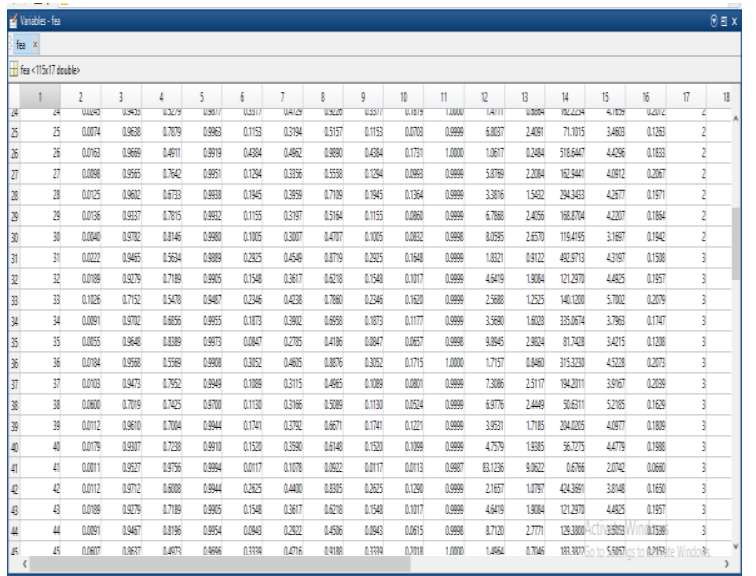

Fig-4: Feature Extraction Result

\section{c) Hybrid Classification}


This paper presents a new method of Hybrid classification algorithm is an extension of $K$ Nearest Neighbor (KNN) and Support Vector Machine (SVM) Classification. A SVM (Support Vector Machine) method works on the principle of structural risk minimization in order to find the best hyper plane that separates 10 classes ( 1 for Beau's lines, 2-Black Nails, 3-Dark reddish,4-greenish, 5-half-white nail, 6lunulae-7-pinkish,8-vertical line, 9-white nails and 10white patches). The SVM is executed on the training image feature instances to attain the reduced Support Vectors (SVs) for every of the sample classes. To combine SVM with KNN classification model used for training and testing classification purpose. Meanwhile, this paper presents categorization process done with trained and testing feature with help of Euclidean distance method with appropriate threshold value.

$$
\operatorname{dist}(P, Q)=\sqrt{\frac{\sum_{m=1}^{N}\left(P_{m}-Q_{m}\right)^{2}}{s t d\left(Q_{m}\right)}} \text { eqn. (1) }
$$

Where, $P$ and $Q$ are trained and testing feature vectors. After the SVM process, a K Nearest neighbor classifier (KNN) is used to classify a testing feature, i.e. the mean Euclidean distance among the testing image feature data point to every set of SVs from different groups is calculated and the category with least distance. A finger nail image GLCM trained feature extraction dataset is given; it chooses the $\mathrm{k}$ nearest samples from the classified training data and determines the class considering the most representative samples. The choice of the parameter $k$ $(\mathrm{k} \in N)$ is determined by the user (i.e., $\mathrm{k}=4$ is defined), this option depends on the image data. A $\mathrm{kNN}$ search on the local features of the training set $\left(T_{s}\right)$ is executed. The consequence of such operation is a list of labeled features $l_{i}$ belonging to $T_{s}$ ordered with respect to decreasing values of the similarity $\operatorname{simm}\left(l_{x}\right.$, $\left.l_{i}\right)$. The class label $\operatorname{cl}^{k}\left(l_{x}\right)$ assigned to the trained data by the classifier is the class $c_{j} \in C$ that maximizes the sum of the similarity between $l_{x}$ and the features $l_{i}$, labeled $c_{j}$, in the $\mathrm{kNN}$ results list $\mathrm{k}\left(l_{x}\right)$. Formally, the hybrid classification have to compute a score $\operatorname{sc}^{\mathrm{k}}\left(l_{x}, c_{j}\right)$ for each class,

$$
s c^{k}\left(l_{x}, c_{j}\right)=\sum_{l_{i \in x^{k}(l x): c l(l i)=c_{j}}} \operatorname{simm}\left(l_{x}-l_{i}\right) \text { eqn. }
$$

(2)
Then the predicted label $c l_{k}$ and the confidence $v$ are defined as follows:

$$
\left\{\begin{array}{c}
c l^{k}\left(l_{x}\right)=\arg _{c_{j} \in C}^{\max } s c^{k}\left(l_{x}, c_{j}\right) \\
v\left(c l^{k}, l_{x}\right)=1-\frac{\arg _{c_{j} \in C-c l^{k}\left(l_{x}\right)} s c^{k}\left(l_{x}, c_{j}\right)}{\arg _{c_{i} \in C} \max s c^{k}\left(l_{x}, c_{i}\right)}
\end{array}\right.
$$

\section{Pseudo Code: Hybrid Classification}

Input: $G T H M$ image, Trained feature $T r_{f}$, Test feature $T s_{f}$, Class label $c l$

Output: Classification Result Class $\mathrm{cl}$ (1 for Beau's lines, 2-Black Nails, 3-Dark reddish,4-greenish, 5half-white nail, 6-lunulae-7-pinkish,8-vertical line, 9white nails and 10-white patches)

\section{Initialization}

Step 1: Choose k value.

Step 2: Calculate distance value of trained and test finger nail feature

Step 3: Extract the disease category image feature with appropriate threshold.

Step 4: Calculate disease similarity score using eqn, (2).

Step 5: Calculate predicted disease class label using eqn. (3)

Step 6: Finger Nail disease classification Result

\section{EXPERIMENTAL RESULT}

The experimental result has been calculated the performance of Hybrid Classification algorithm. The results implemented on Intel I5-6500 series $3.20 \mathrm{GHz}$ 4 core processor, 8GB main memory, and runs on Windows 10 operating system, on which MATALB R2018a. In this experimental work is carried for 480 real time thumb finger nail images which are capture in cannon D50 camera and the overall classification accuracy for all 480 images are calculated with image database could be applied to proposed Hybrid Classification with existing KNN, SVM algorithms which can be flexibly configured to forecast the classification accuracy of data base to meet the needs of various test requirements. 


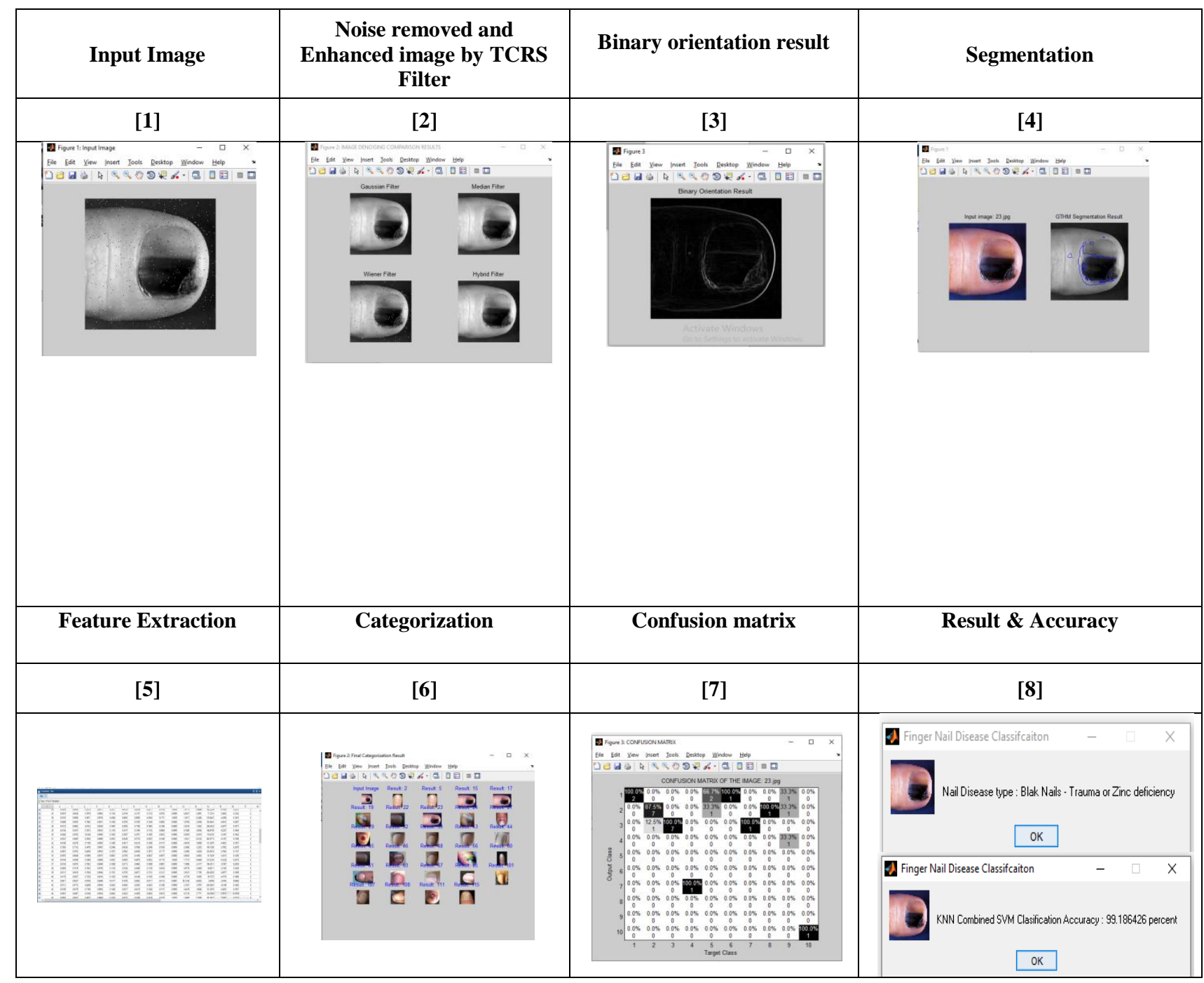

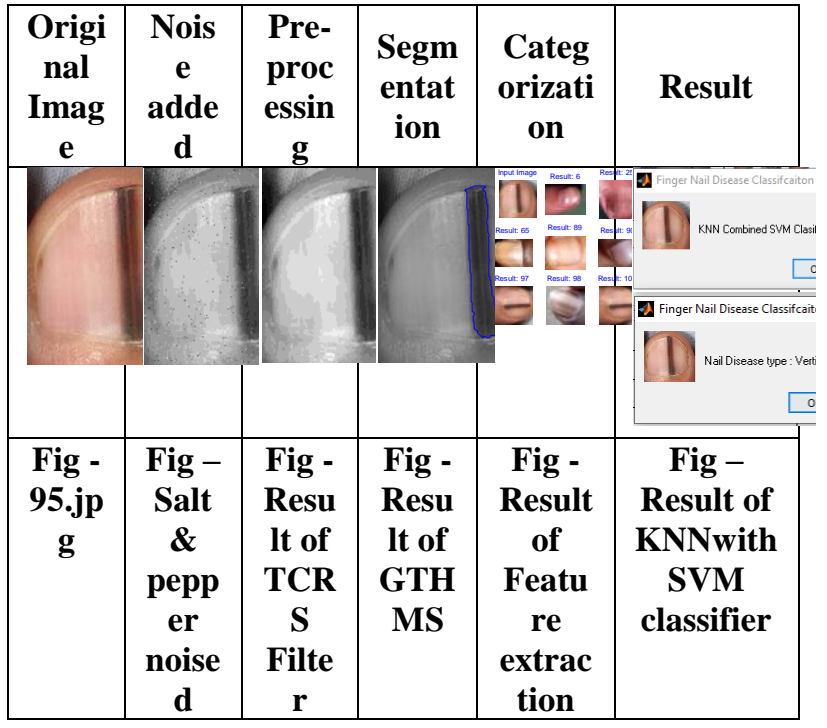

Fig 5: Overall Steps Carried Out By this hybrid classification with Sample Input Image

\section{PERFORMANCE COMPARISON}

Table 1 shows the evaluation of real time finger nail disease database of sample five images of accuracy values for various proposed Hybrid classification with existing KNN and SVM classification algorithms.

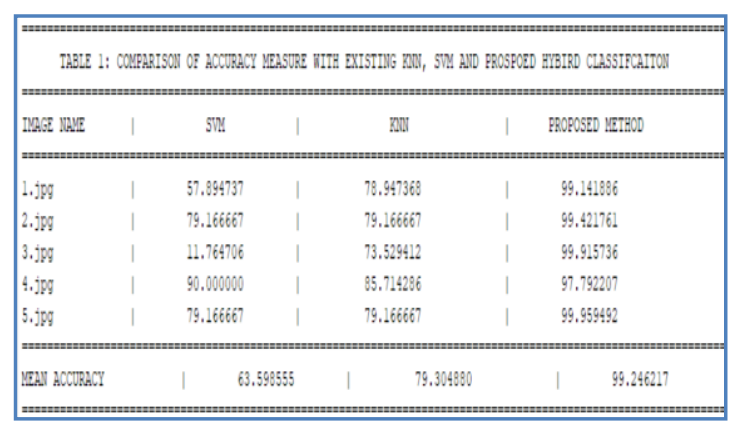




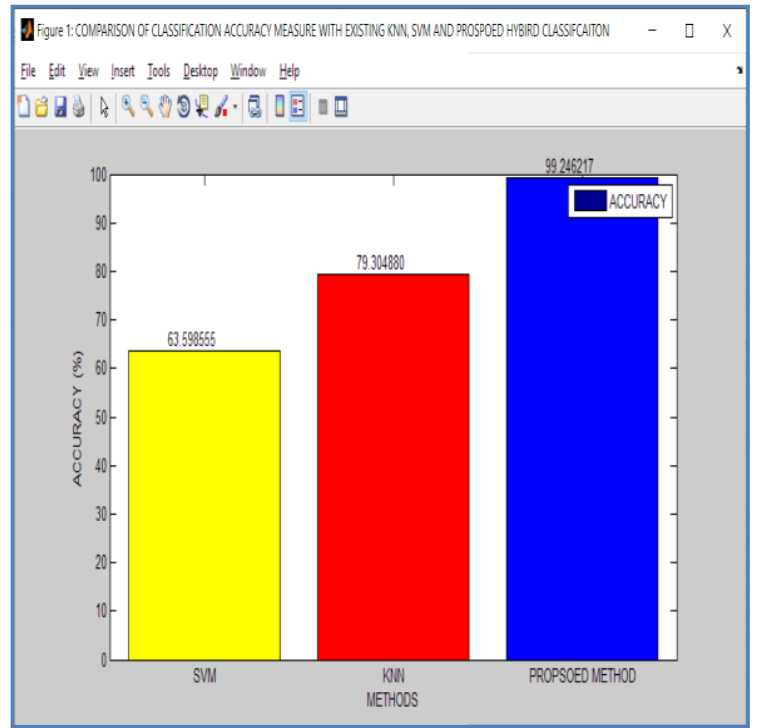

Fig.6: Comparison of Classification Accuracy measures of sample five finger nail images

Table 2 shows the evaluation of Finger nail disease classification measures of accuracy on nail image database. This work is carried for 480 images which are available in finger nail database and the overall accuracy for all 480 images is calculated. The average Classification accuracy of 480 finger nail images and the ratio is defined by,

$$
\begin{aligned}
& \text { OverAll Accuracy } \\
& =\operatorname{abs}(\text { mean(Classifcaiton_Accuracy)) eqn. (4) }
\end{aligned}
$$

Table 2: Comparison of Overall Accuracy of thumb finger nails 480 Images

\begin{tabular}{|c|c|c|c|}
\hline $\begin{array}{c}\text { MEASU } \\
\text { RE }\end{array}$ & SVM & KNN & $\begin{array}{c}\text { HBRID } \\
\text { CLASSIFICA } \\
\text { TION }\end{array}$ \\
\hline $\begin{array}{c}\text { ACCURA } \\
\text { CY }\end{array}$ & $\begin{array}{c}71.10 \\
66\end{array}$ & $\begin{array}{c}80.25 \\
92\end{array}$ & $\mathbf{9 8 . 5 5 4 1}$ \\
\hline
\end{tabular}

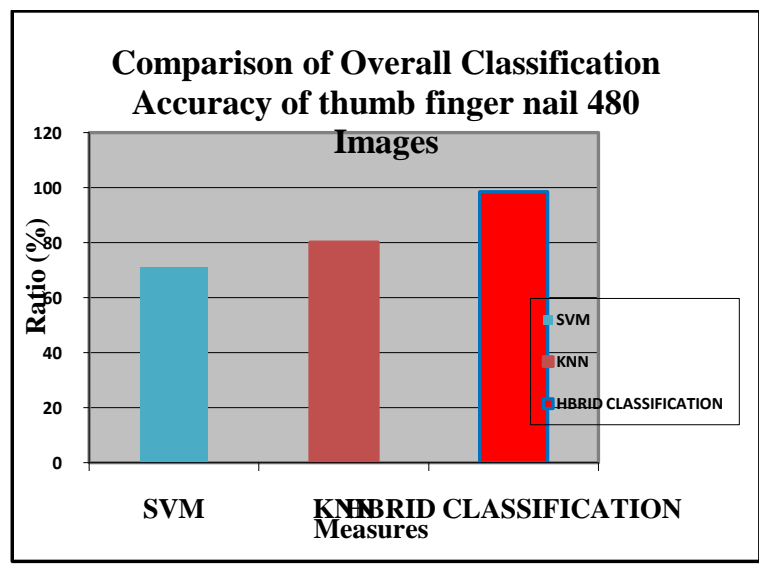

Fig.6: Chart of Classification Accuracy measures of 480 thumb nail images

\section{CONCLUSION}

In this paper, thumb finger nail image hybrid classification methods are examined and the results are shown. Nail abnormalities can be manually evaluated but could not exactly differentiate the colors and textures by all because of the naked eyes. An automated system can ensure error free identification of the abnormalities at lesser time. This paper has proposed a new classifier to find the abnormalities based on the various features that are extracted from the source image. The proposed system uses machine learning algorithms and image processing techniques to segment and classify the images. To overcome the drawbacks of the existing algorithms, TCRS filter, GTHMS-Graph Thresh Histo Match Segmentation and hybrid KNNwithSVM classifier algorithms are used. The hybrid classifier classifies the nail plate abnormalities very efficiently and provides better accuracy than SVM and KNN classifier. The experimental results shows the performance metrics such as classification accuracy with the existing SVM algorithm obtains $71.1066 \%$, KNN algorithm obtains $80.2592 \%$ whereas proposed Hybrid classification yields $98.5541 \%$ overall accuracy.

\section{REFERENCES}

[1] Dr. M.Renuka Devi \& Thahira banu.V, "Study of nail unit using image processing methods", IEEEXplore DOI: 10.1109/ICCCI.2015.7218087, ICCCI Jan 8-10, 2015 (ISBN: 9781479968046)

[2] Thahira banu.V and Dr. M. Renuka devi, "Transitioned Column-Row sorting Filter (TCRSF) To De-noise Finger Nail Images", pages-21-27, volume 11, special issue1, Feb 2019, Journal of Advanced Research in Dynamical and Control Systems

[3] Thahira banu.V and Dr. M. Renuka devi, “GTHM: Graph based Thresh his to match Segmentation (GTHM) In Finger Nail Images", International Journal of Advanced Science and Technology Vol. 29, No. 03, (2020), pp. 7187 - 7197(Scopus Indexed)

[4] Gulnaz Alimjan, Tieli Sun, Hurxida Jumahun, Yu Guan, Wanting Zhou, and Hongguang Sun, "A Hybrid Classification Approach Based on Support Vector Machine and K-Nearest Neighbor for Remote Sensing Data", International Journal of Pattern Recognition and Artificial Intelligence, Vol. 31, No. 10 (2017) 1750034 (22 pages)

[5] Stamatis Gregoriou, George Argyriou, George Larios, Dimitris Rigopoulos, "Nail disorders and systemic disease: What the nails tell us" J Fam Pract. 2008 August;57(8):509-514.

[6] $\mathrm{Dr} K$ Rameshbabu, phanimadhav.yannam, suraj bharath.chada , "Detection of Changes of RADAR Earth Images Using Synthetic Aperture", International 554 
Journal of Engineering Research and Applications (IJERA) ISSN: 2248-9622, Vol. 3, Issue 1, January February 2013, pp.588-593

[7] Sujatha R, Y Sravan Kumar and Garine Uma Akhil, "Leaf disease detection using image processing",
Journal of Chemical and Pharmaceutical Sciences, January-March 2017, JCPS Volume 10 Issue 1, 670672 